Doug Geisler, Eva K. Grebel, and Dante Minniti, eds.

\title{
Modification of the Core Overshoot Treatment
}

\author{
Jong-Hak Woo, Pierre Demaque \\ Yale University, P.O. Box 208101, New Haven, CT 06520-8101, USA
}

Sukyoung Yi

Center for Space Astropysics, Yonsei University, Seoul 120-749 and

University of Oxford, Astrophysics, Keble Road, Oxford, OX1 3RH, United Kingdom

\begin{abstract}
Following Roxburgh's integral constraint, we implemented an upper limit of overshoot in the conventional method of $\alpha$ parameterization in order to remove an overly large overshoot effect for low-mass stars. The erroneously large effect of overshoot due to the failure of $\alpha$ parameterization can be effectively corrected by limiting the amount of overshoot to $15 \%$ of the core radius.
\end{abstract}

\section{The effects of convective core overshoot}

Understanding the physics of convective core overshoot is important in interpreting the color-magnitude diagrams (CMD's) and the luminosity functions of open star clusters, and generally of intermediate-age stellar populations. Convective overshoot is understood as the presence of material motions and/or mixing beyond the formal boundary for convection set by the classical Schwarzschild (1906) criterion. The major role of core overshoot in stellar evolution is to supply hydrogen from mixing in the overshoot region which lengthens the hydrogen core burning lifetime. This results in a much brighter turn off and subgiant branch in isochrones. Also, the luminosity function would be affected by core overshoot since the time scales of each evolutionary stage are modified accordingly ( $\mathrm{Yi}$ et al. 2000). Consequently, if overshoot is significant in stellar evolution, rest frame UV flux would be much higher in the integrated spectral energy distribution of young stellar systems. Thus, in addition to the classical problem of establishing the chronology of star clusters in the Galaxy and nearby systems, core overshoot affects the spectral dating of young and intermediate age galaxies observed at high redshifts.

\section{Test of overshoot treatments}

In stellar evolution calculations, the local pressure scale height is often used to empirically constrain the amount of convective core overshoot. This conventional method assumes that the radial size of the overshoot region is proportional to the pressure scale height at the edge of a convective core. Various observational 

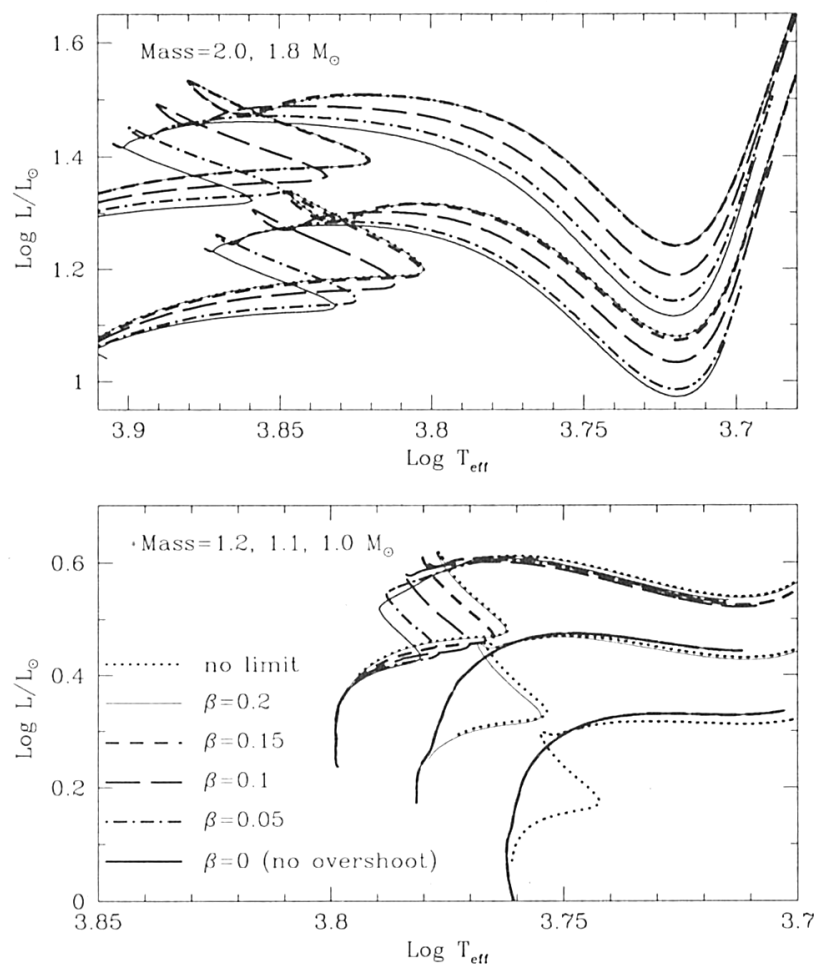

Figure 1. The effect of $\beta$ limit on stellar evoluton for $\mathrm{Z}=0.018$. The amount of core overshoot is limited to $\beta$ factor of $R_{\text {core }}$. Note that models with $\beta \geq 0.15$ show nearly identical tracks for intermediatemass stars (top panel). However, the overshoot effect is not present for low-mass stars when $\beta \leq 0.15$ (bottom panel), which suggests that the erroneously large effect of overshoot on low-mass stars can be corrected by limiting the extent of core overshoot.

studies suggest the amount of core overshoot is around 0.2 pressure scale height (Demarque, Sarajedini \& Guo 1994; Kozhurina-Platais et al. 1997).

However, this method brings unsatisfactory results for low-mass stars which have very small cores or no convective core at all, since the local pressure scale height at the convective core edge would be too large due to the very small core radius. If 0.2 pressure scale height is used as the amount of overshoot, low-mass stars such as 1.0 and $1.1 M_{\odot}$ would show a prominent overshoot effect on their evolutionary shape which is inconsistent with observations. Therefore, simple use of the $\alpha$ parameter cannot give consistent results for a wide range of masses and ages.

In order to remove this artifact, we modifed the conventional method (Woo \& Demarque 2001). The general description of stellar evolution models can be found in Yi et al. (2001). Following Roxburgh's $(1989,1992)$ integral constraint 
which gives an upper limit to the extent of convective penetration, we limited the core overshoot in a way that the amount of overshoot cannot be larger than a portion $(\beta)$ of the core radius.

The effect of the $\beta$ limit is presented in Fig. 1. For low-mass stars, the presence of the overshoot effect is very sensitive to the choice of the upper limit of overshoot. It can be easily noticed that the overshoot effect on low-mass stars (mass $\leq 1.1$ ) becomes negligible for $\beta \leq 0.15$. However, if the upper limit is greater than $20 \%$ of the core radius, the overshoot effect is still too large for low-mass stars. Thus, the erroneously large effect of core overshoot due to the failure of $\alpha$ parameterization can be effectively corrected by limiting the amount of overshoot to $\leq 15 \%$ of the core radius. The choice of $\beta$ does not significantly affect the evolution of intermediate-mass stars $\left(1.5-2.0 M_{\odot}\right)$ when $\beta \geq 0.15$ is used. These models are consistent with those of the same $\alpha$ without limiting the overshoot. However, if the amount of overshoot is restricted to much less than $15 \%$ of the core radius, the overall overshoot effect would be significantly reduced. Therefore $\beta \ll 0.15$ for intermediate-mass stars gives effectively the same result with a smaller $\alpha$ as the conventional method, which is undesirable in our effort to modify the $\alpha$ paramerterizaion with the $\beta$ limit for a given $\alpha$ value. Combining the criteria for low-mass and intermediate-mass stars, we conclude that $15 \%$ of the core radius is a proper limit of overshoot for intermediate to low-mass stars.

\section{References}

Demarque, P., Sarajedini, A., \& Guo, X. J. 1994, ApJ, 426, 165

Kozhurina-Platais, V., Demarque, P., Platais, I., Orosz, J. A., \& Barnes, S. 1997, AJ, 113, 1045

Roxburgh, I. W. 1989, A\&A, 211, 361

Roxburgh, I. W. 1992, A\&A, 266, 291

Schwarzschild, K. 1906, Göttingen Nach., 195, 41

Woo, J.-H. \& Demarque, P. 2001, AJ, submitted

Yi, S., Brown, T. M., Heap, S., Hubeny, I., Landsman, W., Lanz, T., \& Sweigart, A. 2000, ApJ, 533, 670

Yi, S., Demarque, P., Kim, Y.-C., Lee, Y.-W., Ree, C. H., Lejeune, T., \& Barnes, S. 2001, ApJS, accepted (astro-ph/0104292) 